\title{
Evaluation of Distinct Input Methods of an Intelligent Wheelchair in Simulated and Real Environments: A Performance and Usability Study
}

This paper focuses on evaluating the usability of an Intelligent Wheelchair (IW) in both real and simulated environments. The wheelchair is controlled at a highlevel by a flexible multimodal interface, using voice commands, facial expressions, head movements and joystick as its main inputs. A Quasiexperimental design was applied including a deterministic sample with a questionnaire that enabled to apply the System Usability Scale. The subjects were divided in two independent samples: 46 individuals performing the experiment with an Intelligent Wheelchair in a simulated environment (28 using different commands in a sequential way and 18 with the liberty to choose the command); 12 individuals performing the experiment with a real IW. The main conclusion achieved by this study is that the usability of the Intelligent Wheelchair in a real environment is higher than in the simulated environment. However there were not statistical evidences to affirm that there are differences between the real and simulated wheelchairs in terms of safety and control. Also, most of users considered the multimodal way of driving the wheelchair very practical and satisfactory. Thus, it may be concluded that the multimodal interfaces enables very easy and safe control of the IW both in simulated and real environments.

Keywords: Intelligent wheelchair; Multimodal interface; Powered wheelchairs; Adaptability; Assistive robotics; Assistive technology; Human-machine interface

\section{Introduction}

Nowadays the importance given to the autonomy and independence of elderly people and patients suffering from some kind of disabilities is growing. In fact, it was proclaimed by the United Nations General Assembly the rights of people with disabilities in which it is defended that "People with disabilities have the right to the measures to enable them to become as independent as possible" and "People with disabilities are entitled to medical treatment, psychological and functional treatment, including prostheses and orthoses, rehabilitation and social medical treatment..." 
(Byrnes, 2007). Another group that is growing and needs substantial attention is the elderly people. The number of elderly individuals and the rate of growth of the elderly population are increasing in almost all developed countries (UN, 2002). So it is important to give an alternative that allows the elderly and the handicapped to be as autonomous as possible. Wheelchairs are one of the main tools to achieve this higher degree of autonomy. As it was defended by Vignier et al. (Vigner et al., 2008) the dependent elderly are the most common candidates for wheelchair and a larger number of wheelchair users live in institutions. Moreover the wheelchairs are evolving to Intelligent Wheelchairs which can further help elderly and people with disabilities like Alzheimer, Parkinson and Cerebral Palsy (Simpson, 2008). Innovations in health sciences are increasingly related with this kind of new intelligent devices and techniques provided by the engineering community and with its correct use to aid different groups of patients.

A wheelchair may be seen as a wheeled device that may be propelled either manually or using motors. Wheelchairs are instruments that were initially developed in order to give mobility to handicapped human beings. Currently the wheelchairs are seen as powerful resources to overcome more severe limitations and disabilities resulting from several types of handicaps and illnesses. Moreover the concept of intelligent wheelchair (IW) is a natural development of the scientific work that has been conducted to improve the traditional Wheelchair characteristics. Some of the most important features of the IW are their navigation capabilities and automatic adaptability of their interface to the user (Braga et al., 2009) (Reis et al., 2010).

The concept evolved from simple manually powered wheelchairs to electric wheelchairs and today new developments are presented in so called intelligent 
wheelchairs or "smart chairs" or even "robotic chairs" (Simpson et al., 2004) ( Simpson et al., 2005) (Cooper et al., 2010).

The first intelligent chairs were basically typical mobile robots to which seats, capable of accommodating a user, were added (Simpson et al., 2005). Nowadays, science allows having intelligent wheelchairs, very similar in shape to traditional wheelchairs, with high manoeuvrability and navigational intelligence, with units that can be attached and/or removed and with high power independence.

\section{Definition and characteristics of an Intelligent Wheelchair}

Definitions of Intelligent Wheelchair can be found at (Simpson et al., 2005) (Braga et al., 2009) (Reis et al., 2010). Basically, an IW is a locomotion device to assist a user having some kind of physical disability, where an artificial control system augments or replaces the user control (Simpson et al., 2004). The main objective is to reduce or eliminate the user's task of having to drive a motorized wheelchair. Typically, an IW is controlled by a computer, it has a set of sensors and applies techniques derived from mobile robotics research in order to process the sensor information and generate the motors commands. The interface may consist of a conventional wheelchair joystick, voice based control, facial expressions (Faria et al., 2007) or even vision control, among others. The concept of IW is different from a conventional electric wheelchair, since in this latter case the user takes manual control over motor speed and direction via a joystick or other switch, without intervention by the wheelchair's control system. The main characteristics of an IW are (Braga et al., 2009) (Jia et al., 2007):

- Interaction with the user using distinct types of devices such as joysticks, voice interaction, vision, pressure sensors or other sensors;

- Autonomous navigation with safety, flexibility and obstacle avoidance 
capabilities;

- Communication with other devices such as automatic doors and other wheelchairs.

\section{Intelligent Wheelchairs' Projects}

Although there are several projects in the area of intelligent wheelchairs and some business models that use new information technologies and robotics in support of a profound disability, there is a lack of wheelchairs with real capabilities of intelligent actions planning and independent navigation. Many Intelligent Wheelchair prototypes are being developed in several research projects around the world, however the adaptation of their user interface to the patients' individual characteristics is an often neglected research topic. Typically the interfaces are very rigid and adapted to a single user or user group (Mason et al., 2010). Many prototypes have been used to explore a variety of alternatives to the more traditional input methods associated with powered wheelchairs such as joystick. Voice recognition has often been used for intelligent wheelchairs such as NavChair (Mason et al., 2010), RobChair (Pires et al., 2002), SENARIO (Katevas et al., 1997), Tetra-Nauta (Vicent et al., 2002), because of the low cost and widespread availability of commercial voice recognition hardware and software. More exotic input methods that have been implemented include the detection of the wheelchair user's sight path, namely where the user is looking through electrooculographic (EOG) activity such as Wheelesely (Yanco, 1998) or the use of machine vision to calculate the position and orientation of the wheelchair user's head as it was proposed by Matsumoto et al. (2001). Some more recent projects presented input methods for people with quadriplegia, by using the recognition of facial expressions as the main input to guide the wheelchair. Recently, researchers at the Georgia Institute of Technology presented a prototype of a tongue mouse that may use by individuals with 
spinal injuries as a joystick to control motorized wheelchairs (Vogel, 2008). Another solution suggested by a group of researchers consists of operating an intelligent wheelchair using the thought as an input method, by means of sensors that capture the user's brain electromagnetic waves.

\section{Intelligent Wheelchair's Interface Adaptation}

Most of the wheelchair projects presented (Simpson et al., 2004) did not include any reference to the user adaptation to the wheelchair and how to improve the IW interface based on the user interaction with the device. Therefore, an important part of the study is the interaction of the users with the IW and how an intelligent/adaptive interface can help and improve the user mobility. The interface between a human and a computer is called a user interface and it is a very important part of any computerized system. Moreover an adaptive user interface (Langley, 1999) is a software entity that improves its ability to interact with a user by constructing a user model based on experience with that user. The emerging area of adaptive and intelligent user interfaces has been exploring applications in which these paradigms are useful and facilitate the human machine communication (Ross, 2000). In fact, if an intelligent user interface has a model of the user, then this user model can be used to automatically adapt the interface. Furthermore, adaptive user interfaces may use machine learning to improve the interaction with individuals in order to have the users reach their goal more easily, faster and with a higher level of satisfaction. It is also essential for an adaptive interface to obtain knowledge included in four distinct domains: knowledge of the user; knowledge of the interaction (modalities of interaction and dialogue management); knowledge of the task/domain; and knowledge of the system characteristics (Norcio et al., 1989). 
Other concepts concerning adaptive interfaces that must be considered are the usability, accessibility and safety. In fact, almost every investigation of intelligent wheelchairs reports brief studies of usability and accessibility. Recently an interesting study about the usability of a multimodal feedback interface was published (Wang et al., 2011). This study concerns with the response of a power wheelchair with obstacle avoidance to a user with cognitive and complex physical impairments.

\section{IntellWheels Project}

Intellwheels project is aiming at developing a new concept of Intelligent Wheelchair. The prototype is controlled using high-level commands, triggered using combinations of distinct inputs in the context of a multimodal interface. Another characteristic of the IntellWheels Project is to develop an intelligent wheelchair platform that can be adapted to any electrical commercial wheelchair and aid any person with special mobility needs (Braga et al., 2009) (Braga et al., 2011). Several different modules have been developed in order to allow different ways of conveying commands to the IW. There are already several commands implemented, such as joystick control with USB, voice commands, control with head movements. The system allows users to choose which type of command best fits their needs, increasing the level of comfort and safety.

Currently these commands are implemented in a prototype composed by an electrical wheelchair, a personal computer with software specifically created, a monitor with the multimodal interface (Vasconcelos, 2011), sensors and actuators. Figure 1 shows the real prototype of the intelligent wheelchair.

However, in order to fully control the wheelchair, the user must have a Wheelchair interface adapted to his characteristics. And for that, the system (wheelchair and/or user) must be able to understand what exactly the user's characteristics are 
concerning interacting with the wheelchair. It was also developed IntellWheels Simulator (Braga et al., 2010). IntellWheels Simulator is a tool that creates a virtual Intelligent Wheelchair, identical to the current IW prototype, which can be controlled using all interaction modes of the real prototype. The objective is to conduct similar experiments as in real environment, but with low cost, since it is easier to obtain results in the simulated environment.

\section{Objectives}

The main objectives of this investigation were threefold: to determine the usability of the IW, to evaluate the usefulness of different IW commands in real and simulated environment and to verify if there are statistical differences between the experiments in the two environments. The hypothesis of study is: The usability, safety, control and performance of the IW are identical in real and simulated environments. In fact the ultimate objective is to use the simulator to train potential users of the IW.

\section{Methods}

The potential and final users of IW are handicapped individuals and patients with distinct disabilities like Thrombosis, Stroke, Cerebral Palsy, Parkinson, Alzheimer and Multiple Sclerosis. The population of this study is composed of individuals that are more sensitive to typical patients' problems. This means that the population of this study had previous contact and studied the problems of the potential users of the IW. In fact, since this is a preliminary study of the usability of the IW, it was established at this first stage to perform the tests both in simulated and real environments to check the possibility of using the simulated environment to train the future users of the IW. 


\section{Participants}

The results evaluation was based on empirical research and involved 46 students from Physiotherapy in the School of Allied Health Sciences of Porto. The individuals were initially divided into four groups with 14 elements. Two groups of 14 elements (making a total of 28) used all four input methods for driving the IW in a sequential way. The other two groups had indications to choose any kind of command but on these groups only 18 out of the 28 elements performed the tests. This kind of analysis is very important since this type of population, due to their background, is more sensitive for the questions of Intelligent Wheelchairs' usability. Thus it enables more easily future applications to handicapped individuals. A second experiment, involved a sample of 12 volunteers that performed a similar test but with a real IW in a real environment. The individuals of the sample were recruited in collaboration with a joint communitarian project which it is part of the curriculum of the School. The review committee of the School of Allied Health Sciences of Porto was created only after this study. We obtained authorization of the responsible professors and from all the individuals that participated in these experiments.

\section{Instruments}

The methodology applied was the gathering of opinions using a questionnaire that incorporates the System Usability Scale (SUS) (Brooke, 1996) which is a simple ten-item Likert scale (Field, 2005) giving a global view of individual assessments of usability (Brooke, 1996). The questionnaire was divided into 3 parts:

- User identification - Several questions about gender, weight, height, level of education and experience of using video games and appropriate commands;

- Usability - The System Usability Scale was applied; 
- Safety and Controls of the IW - Questions about control, attention and safety feeling when driving the IW were asked. A general question on the level of satisfaction with different command modes of the IW, such as joystick in manual and high level modes, voice commands, head movements and integration of all kind of commands was also asked.

The type of study can be classified as quasi-experimental design in which the kind of sampling used was deterministic.

\section{Procedure}

In order to obtain the feedback of the IW two major experiments were performed. The first one was organized in the School of Allied Health Sciences of Porto. A simulated scenario of part of the School was created with a desired route marked in it that should be followed by the users driving the wheelchair. Figure 2 shows a 2D image of the complete tour and the first person view of the 3D simulator.

The average error of deviation was only possible to calculate in the simulated environment. It was calculated as the average distance from the point of the real trajectory to the "ideal" trajectory (the IW should follow in the middle of the corridor). In fact, the distance of the IW to the nearest segment of the "ideal" trajectory was calculated in every simulation cycle, and the sum of this value was divided by the number of cycles. The distance from this trajectory was given using a simple algorithm to calculate the distance from a point to a line segment (Math, 2010) (Okabe et al., 2000). The length of the total route of the "ideal" trajectory was 51.5 units. To make this route several scenarios were defined, where the user could drive the IW:

- using the gamepad joystick in manual mode (the commands are directly send to the motors and actuators of the IW); 
- using the gamepad buttons in high-level mode (by pushing the buttons different actions triggered);

- with head movements (using accelerometer from the wii controller);

- with voice commands;

- having the freedom to choose any type of input (gamepad, voice, head movements).

Twenty eight users made the experiment using all IW driving modes, in a sequential way: joystick in manual mode, gamepad in high-level, head movements and voice commands. However, only 18 individuals used the IW having freedom to use any of the available commands. The order of the input was initially set randomly. Everybody performed the experiments in the same order (to avoid extra elements of distortion in the results). The experiments were performed disabling the smart wheelchair obstacle avoidance module in order to be able to measure the number of collisions since this is a good method to evaluate the users' driving abilities.

These scenarios were conducted in a 3D simulator. This simulator was developed and it allows to test control algorithms and to simulate the physical behaviour of the intelligent wheelchair (Braga et al., 2008) (Braga et al., 2010). This simulator was originally based on the Cyber-Mouse (Lau et al., 2002) simulation system. This simulation system is able to represent a virtual area of a building, identical to a hospital or other type of space, with several areas that represent bedrooms, a kitchen, a bathroom, a hall, a garden, among others, populated with obstacles. The simulator can simulate all the sensors and actuators of the IW and can manage the movement of several IW within that virtual environment.

This type of platform has two main objectives. One is the creation of a virtual test based control and interaction of wheelchairs. The other objective is to test the same 
interaction between real and virtual chairs. This system allows testing a single wheelchair or multiple wheelchairs in the context of a very complex system, without constrains of the number of real prototypes available.

The second experiment was performed by 12 volunteers in a similar circuit however in the real environment. The floor was marked and the individuals should drive the IW also in the middle of the corridor. The real IW also incorporates a Multimodal Interface in a small monitor as can be observed in Figure 3.

The proposed multimodal interface offers three basic methods of recognition: speech recognition, recognition of head movements and the use of a generic gamepad. In addition, it is proposed an architecture that makes the interface extensible at this level, making it possible to add new devices and recognition methods in a transparent approach. It also presents a flexible paradigm that allows the user to define the sequences of inputs to assign to each action, allowing for an easy and optimized configuration for each user.

Since none of the individuals had any type of previous contact with the IntellWheels project, the first step, in both experiments, was to provide an explanation of the main characteristics of the IntellWheels Multimodal Interface, the type of output actions provided by the IntellWheels Platform control module, and the global goals of the experiment. Also, we had to explain the set of different input sequences that were prepared for this experiment. Table 1 contains the defined input sequences. Before the controlled experiments were recorded the individuals had the opportunity of driving the wheelchair using different commands.

The data analysis was performed using the following software: the IBM SPSS Statistics Standard (version 18.0), Excel 2007, Dev C++ in order to calculate the distance of the point to a line segment, G*Power and PASS11 (Hintze, J., 2012) to 
calculate the power of the statistical tests. The statistical tests applied were the ShapiroWilk for the normality, the Student's t-test for comparing independent samples and the Mann-Whitney to compare distributions. In order to test the statistical significance of the performance differences between the used commands of the IW, the Friedman test was applied. To identify which commands are significantly different it was necessary to use a multiple comparison of means of orders (Fisher's least significant difference (LSD)). The level of significance used was of 0.05 .

\section{Results}

The results are organized with the samples characterization, the usability results, the safety and control of the IW and with the performance of driving the wheelchair. The samples characterizations were performed by describing demographical information and individuals' experience by using videogames and gamepads. The usability was achieved by the score of SUS and a test to compare the two independent samples was realized. The safety and control of the IW questions intent to reveal the individuals' feelings in terms of control and safety by driving the wheelchair and been far from any wall or obstacles. Finally the time, number of collisions and distance from the desired trajectory served to obtain results about the performance of driving the IW.

\section{Samples' characterization}

In the first experiment the dimension of the sample was of 46 individuals, with a mean age of 21 years old and standard deviation of 2.23. The sample is also characterized by having a larger number of females: 42 females and only 4 males. All of the individuals are students of the 3rd year of Physiotherapy at School of Allied Health Sciences of Porto and did not present any kind of disabilities. In order to test the performance of the real IW a sample of 12 volunteers without any disabilities (in which 3 performed the 
two experiments) made a similar tour using the real IW. The mean of age was 23 years old with a standard deviation of 5.99.The Table 2 also presents the mean, the standard deviation, minimum and maximum of weight and height of the samples. It was important to know beforehand the experience of the users using videogames and gamepads, since the IW multimodal interface offers this type of control devices. Most of the answers focus on Never or Rarely noticing the lack of experience with this kind of devices of the individuals.

\section{Usability of the IW}

In terms of usability, the SUS scale score is a measure that can be easily interpreted. The value varies from 0 to 100 and if the value is near 100 it means that the individual considered the instrument extremely effective, efficient and satisfactory. In this experiment the result of the total score has a mean of 63.3 and a standard deviation of 15.5 . The median is 66.3 , the mode 75.0 , the minimum is 20.0 and the maximum is 87.5.

The total score of SUS in the real experiment has a mean of 77.0 and a standard deviation of 12.4. There were three individuals that participated in both experiments and the total score of SUS increased from the first experiment (using a simulated environment) to the second one (using a real wheelchair) except in one case. In fact, the result of the real IW experiment was slightly conditioned by some problems of the IW firmware that made the IW turn off sometimes during the tests (having to be quickly restarted to continue the experiment). The internal consistency reliability was also measured and the Cronbach's Alpha ascended to 0.837.

So the next step was to understand if there were differences between the results of usability in simulated and real environments. The normality test Shapiro-Wilk was applied for this and the $\mathrm{p}$ value achieved $(0.119$ in the case of the group that use real 
IW) was bigger than the level of significance (0.05). Therefore the normality of the data can be assumed and, thus, the suitable test to perform this analysis was the two independent sample $t$ test (in order to check if there were significant differences in the means in those two cases). The $p$ value of Levene's test (G*Power, 2010) for equality of variances was 0.548 (thus, higher than the level of significance). For that reason the variances can be assumed as equal.

Finally the $\mathrm{p}$ value of the two independent samples t test was less than 0.005 (thus, less than the significance level of 0.05). This enables us to conclude that there are statistical evidences to claim that the mean of the total score of SUS in the experiment with the simulator is different from the mean of the total score of the SUS in the experiment with the real IW. This way, we may claim that the mean of the total score of SUS is higher in the experiment with the real IW than using the simulator. The power achieved with this test was approximately 0.5 with an effect size of 0.5 .

\section{Safety and Control of the IW}

Some questions about safety and control were also proposed and the results can be observed in Figure 4 for the case of simulated and real environments. From these results it is very interesting to conclude that they were very similar for the simulated and real environments. Applying the Mann-Whitney test in order to compare the distribution of the answers about safety and control it is possible to conclude that there are not statistical evidences to affirm that the distributions are not identical. In fact the $\mathrm{p}$ values of all questions related to safety and control are higher than the significance level of 0.05: I felt safe in the management of IW (0.420); I felt I had control of the IW (0.223); It is easy to drive in narrow places $(0.058)$ and IW does not need too much attention (0.359). The power achieved was 0.32 with an effect size of 0.5 . 


\section{Performance of driving the IW}

The performance achieved by using the different modes for driving the IW were assessed by the time to complete the path, number of collisions and average distance from the real trajectory to the desired trajectory.

The time spent in order to complete the circuit was measured using a chronometer. In the simulated environment three users had deviations from the original route and became lost in the circuit: one element from the group of people that made the experiment using all the simple driving modes and two users that had the liberty of using the available commands. So, their results had to be eliminated from the performance's statistical analysis. Users which performed the experiment with voice commands spent, in average, a higher time $(6.70 \mathrm{~min})$ to complete a tour than those using other command modes. The shorter time average was achieved using head movements (3.80 min). However, the shorter for the median was achieved using the gamepad with high-level commands (3.27 min). This last command mode presents the highest dispersion with a standard deviation of $1.29 \mathrm{~min}$, a minimum of 2.88 and a maximum of $11.65 \mathrm{~min}$. It seems to be a very effective mode for driving the IW for users that are able to correctly use it but it is also a very ineffective mode for a small number of users. The performance in terms of time having the freedom to drive with all commands did present values near to others methods of manoeuvring the IW. The standard deviation in this case was the shortest with a value of 0.97 . Also the maximum achieved for this mode was the shortest of all. Applying the Friedman's test to the paired samples of using the four commands of driving the IW a p value of 0.002 was obtained. So there are statistical evidences to affirm that at least one of the commands has a different distribution of time. The power achieved was 0.706 with an effect size of 0.5. The $\mathrm{p}$ values of the multiple comparisons, using the Fisher's least significant 
difference (LSD), can be observed in Table 3. The statistical differences occur between the time taken when using voice commands and the time taken for the rest of the commands.

In the real environment the higher average time was also spent using voice commands (6.33 min). The shorter average time was achieved using head movements (4.36 min). The average time using the gamepad and the joystick were 5.33 and 4.38 min respectively. The standard deviation was similar $(1.4 \mathrm{~min})$ in the head movements and joystick manual modes. The lowest value was presented by the high level joystick mode with 0.7 min. Applying the Friedman test in this case the $\mathrm{p}$ value obtained was less than 0.001 ( $\mathrm{p}$ values of the multiple comparisons are presented in Table 3). The power achieved applying this test is approximately 0.34 with an effect size of 0.5 . The conclusions are similar (to the simulated environment) in what concerns the time spent using different command modes in the real environment.

Table 4 contains the number of users that made the experience in different conditions and the measures of central tendency (mean and median) and dispersion (standard deviation, minimum and maximum) of the number of collisions in the experiment using the simulator and the real IW.

Analysing the best performance in terms of collisions in the simulated environment, the gamepad at high-level presented clearly the best average (3.33 collisions) and the voice commands also presented the worst average of collisions (31.83) confirming the worst result achieved for the time measure using this command mode. The $\mathrm{p}$ value of the Friedman's test (less than 0.001 ) provides evidence that at least one of the commands has a different distribution of collisions. The power achieved was 0.706 with an effect size of 0.5 . The $p$ values of the multiple comparisons, using the Fisher's least significant difference (LSD), can be observed in Table 3. The statistical 
differences occur between the numbers of collisions when using voice commands and when using the rest of the commands. In the real environment the number of collisions was also quantified. These results express a lower number of collisions in real environment and the behaviour is almost identical when using the different commands. Confirming with the Friedman's test the $p$ value was 0.001 . The $p$ values of the multiple comparisons are presented in Table 3 . The power achieved applying this test is approximately 0.34 with an effect size of 0.5 .

Table 5 has the values of the average error of deviation from the "ideal" trajectory which was asked to users to follow. The average error of deviation from the trajectory does not present major differences between the different modes for driving the IW (Friedman's test $p$ value $=0.659)$. However, it is important to remark the measures of dispersion in the case in which the users had the freedom to choose any type of command. In fact, the standard deviation has the highest value and the difference between the minimum and maximum is also the highest. This reveals that in this mode, the individuals were more distant from the "ideal" trajectory and less homogeneous, comparing to the other command modes.

Figure 5 shows the trajectories obtained by one of the users in a lap using the different driving modalities. As it can be seen, there is a certain consistency in the trajectories obtained for the first three modalities. In this particular case, the attendant had some difficulties to control the wheelchair at a certain stage of the route, using voice commands. In spite of the different performances and trajectories obtained by each of the individuals, this example illustrates the global tendency.

One other aspect that was analysed was the level of satisfaction about the different commands as it is depicted in Figure 6. Clearly the answers of satisfaction with the commands given by the individuals that made the experiment with the real IW are 
more positive satisfactory than those who made the experiment in the simulated environment. It was applied the Mann-Whitney test to verify if there were statistical differences between the answers to the corresponding questions related to commands used in real and simulated environments. The $\mathrm{p}$ values of the question about the degree of satisfaction with joystick in manual mode and voice commands were higher than the level of significance, respectively 0.464 and 0.845 . The $p$ values of the question about the degree of satisfaction with joystick in high level and with head movements were respectively less than 0.001 and 0.023 . Hence, it is possible to affirm that there are statistical evidences that the distribution of the answers in these two last cases is significantly different in real and simulated environments. The power was of 0.32 with an effect size of 0.5 .

\section{Discussion}

The IntellWheels project ultimate goal is tackling the problem of user interface adaptation to distinct patients by defining several options for driving an IW and integrating all in a multimodal interface. For that it is necessary to obtain information about the behaviour of individuals in a simulated environment, in comparison with the real environment, since data collection is easier using the simulator than using the real IW. Besides enabling easier and more precise data gathering, the simulated environment allows: higher control with lower costs, easy comparison of alternative designs or operating policies, sensitivity analysis, a very good training tool, does not have the problem of being susceptible to damage by inappropriate use and enables easy replication for conducting parallel experiments. By performing tests in real and simulated environments the principal findings were that the mean of the total score of SUS is higher in the experiment with the real IW than using the simulator. This result 
was expected since the users are less familiar with the simulated environment and also its reduced realism seemed to be a limitation for achieving a higher SUS score.

In this study there were not statistical evidences to affirm that the distributions are not identical in terms of safety and control in the real and simulated experiments. However, in the case of the answers to the statement "It is easy to drive in narrow places", the obtained $p$ value was 0.058 , which reveals a trend towards statistical significance of the difference of difficulty for driving the IW in narrow places. In these cases the power of statistical tests obtained was low with a medium effect size which reveals limited ability to detect the differences. Thus, to overcome this constraint concerning the low statistical tests' power, in future work, it may be important to perform tests in the real environment with a bigger sample. Other performance measures were accessed by the number of collisions, time consumed and average deviation from the "ideal" trajectory. It is important to emphasize that the ideal trajectory was initially defined and the users were informed that the objective was to follow the route as close as possible of the middle of the corridors. The deviation allows verifying the accuracy in following a pre-defined route. The results show statistical differences between the time and collisions when using voice commands, compared with using any other of the other available commands, in both environments. In real environment there are also statistical evidences to affirm that the number of collisions is different between the head movements and the high level joystick mode. For the simulated environment, the test power achieved on this analysis can be classified as good, considering the predetermined level of significance, and the effect size may be classified as medium. By individually comparing the efficiency of each input module, we discovered that the majority of individuals preferred using the gamepad to introduce high-level commands. However, some preferred other types of interaction, such as using 
the head to control the wheelchair. Although substantially smaller, a number of participants selected the voice commands as being a trust worthy style of driving the IW. In fact, controlling the wheelchair using voice commands represented the most difficult part of the challenge. After this experiment, we conclude that the length of the voice commands should be as short as possible. For example, instead of having the voice command "Turn Right", we could use an alternative expression: "Right" or even a shorter command. This would decrease the delay time between the intention of turning right, and the actual output action "Right Turn" triggering. Considering the size of the room where these experiments were performed, and the characteristics of the proposed route, which was difficult and narrow, the obtained results were very satisfactory. Probably using voice commands in wide spaces the results could be better, since the time between the command and the IW action has some delay. Moreover, it is important to refer the lack of experience and training level of most of the volunteers, and the fact that the wheelchair's obstacle avoidance feature was not used during the experiment. Thus, we believe that combining good user training with the wheelchair's capacity to avoid obstacles is enough to provide a safe and reliable voice command experience. Also, this command mode may be one of the few, or even the only one, that very handicapped quadriplegic users (those are even unable to perform head movements) may use to command the wheelchair.

The main hypothesis stated on this study was formulated as: "The usability, safety, control and performance of the IW are identical in both real and simulated environments". The statistical results showed, as discussed in the previous paragraphs that the usability of the IW in the real environment was slightly higher than in the simulated environment. The performance of the individuals in terms of collisions can help us understand this conclusion. In fact, the number of collisions was different in real 
and simulated environments especially when using the voice commands. We noticed that a stronger effort was put by the participants in order to avoid collisions using the real IW. This result also raises the importance of the adaptation of the interface to the user. For example it is possible for a given individual to drive an IW using voice commands without any collision, but for some other individual this kind of command may be very difficult to use. The assessment of safety and control did not reveal significantly statistical differences between real and simulated environments confirming the initial hypothesis.

The limitations of this study, that should be tackled in the future, are mainly concerned with the necessity of using validated instruments to further validate the user satisfaction with the proposed assistive technology and use a bigger sample of individuals in the real environment.

This study tested the multimodal interface developed in order to prepare its complete test with real patients. The used samples were composed with individuals that are more conscious about the problems of the potential users of the IW. However this is a limitation of our study, because the ultimate goal is to perform experiments with real handicapped people. Nevertheless these experiments allow concluding that it is possible and useful to drive the IW using a multimodal interface.

\section{Conclusions and Future Work}

The IntellWheels project aims at developing a new concept of Intelligent Wheelchair controlled using high-level commands integrated in a multimodal interface. However, in order to fully control the wheelchair, the user must have a wheelchair interface adapted to his characteristics. Thus, the pertinence of this study comes to overcome the lack of information about users' characteristics concerning their 
wheelchair command preferences. It enabled to get more experience and knowledge about new interfaces and commands for Intelligent Wheelchairs.

The study enabled us to have results on the multimodal way of driving the intelligent wheelchair. In terms of usability, safety and control of the IW in simulated and real environments the results are practical and satisfactory. Also, the usability of the Intelligent Wheelchair in a real environment is higher than in the simulated environment showing the usefulness of the real prototype developed.

For future work it is intended to apply Machine Learning techniques for automatic patient classification. This will enable us to adapt, in a straightforward manner, the flexible IW multimodal interface to each user, highly improving its usability. Work is already being developed to improve the realism of the simulator, adding for example dynamic aspects, as well as to shorten the gap between virtual and real realities. The tests with real handicapped people will be performed in near future.

\section{References:}

Braga R., Petry M., Moreira A.P., Reis L.P. (2009). Concept and Design of the Intellwheels Plataform for Developing Intelligent Wheelchairs. Springer-Verlag, LNEE/ Informatics in Control, Automation and Robotics, 37, (pp. 191-203).

Braga, R., Malheiro, P., Reis, L. P. (2010). Development of a Realistic Simulator for Robotic Intelligent Wheelchairs in a Hospital Environment. RoboCup Symposium 2009, Springer LNCS, 5949, (pp. 23-34). Graz, Austria.

Braga, R., Petry, M., Reis, L.P., Moreira, A.P. (2011). IntellWheels: Modular development platform for intelligent wheelchairs. Journal of Rehabilitation Research \& Development; 48(9): 1061-1076.

Braga, R., Petry, M., Reis, L.P., Oliveira, E. (2008). Multi-level control of an Intelligent Wheelchair in a Hospital environment using a Cyber-Mouse Simulation System, in 5th International Conference on Informatics in Control, Automation and Robotics, Vol. II, (pp. 179-182). Funchal, Portugal. 
Brooke J. (1996) SUS: A quick and dirty usability scale. In P. W. Jordan, B.

Weerdmeester, A. Thomas, and I. L. Mclelland, editors, Usability evaluation in industry, London, Taylor and Francis, (pp. 189-194).

Byrnes A. (2007). Disabilities: From Exclusion to Equality. Geneve: United Nations Office of the High Commissioner for Human Rights, 2007.

Cooper R.A., Cooper R., Boninger M. L., (2008). Trends and Issues in Wheelchair Technologies. Assistive Technology, 20, 2, 61-72.

Faria P.M., Braga R., Valgode E., Reis L.P. (2007). Interface framework to drive an intelligent wheelchair using facial expressions. IEEE International Symposium on Industrial Electronics (pp. 1791-1796). Vigo.

Field, A. (2005). Discovering Statistics using SPSS, Second Edition, SAGE Publications, London.

G*Power, Institut fur Experimentelle Phycologie, G*Power 3, (2010). Retrieved July, 30, 2011, from http://www.psycho.uniduesseldorf.de/abteilungen/aap/gpower3/.

Hintze, J. (2012). PASS 11. NCSS, LLC. Kaysville, Utah, USA.Retrieved January, 20, 2012, from http://www.ncss.com.

Jia P., Hu H., Yuan K., (2007). Head Gesture Recognition for Hands-free Control of an Intelligent Wheelchair, Industrial Robot: An International Journal, 34, no. 1: 6068.

Katevas, N.I., Sgouros, N.M., Tzafestas, S.G., Papakonstantinou, G., Beattie, P. (1997) The autonomous mobile robot SENARIO: a sensor aided intelligent navigation system for powered wheelchair. Robotics \& Automation Magazine, IEEE. 4: 6070.

Langley P., (1999). User modelling in adaptive interfaces, Proceedings of the 7th international conference on user modelling, (pp. 357-370). Banff.

Lau, N., Pereira, A., Melo, A., Neve A., Figueiredo, J. (2002). Ciber-Rato: Uma Competição Robótica num Ambiente Virtual. Ciber-Mouse: One Robotic Competition in a Virtual Environment. Magazine of DETUA, 3, no. 7, 647-650.

Levine S.P., Bell D.A., Jaros L.A., Simpson R.C., Koren Y. (1999). The NavChair assistive wheelchair navigation system. IEEE Transactions on Rehabilitation Engineering, 7, 443-451.

Mason B.S., Porcellato L., van der Woude L.H.V., Goosey-Tolfrey V.L. (2010). A Qualitative examination of wheelchair configuration for optimal mobility 
performance in wheelchair sports: A pilot study. Journal of Rehabilitation Medicine; 42, 141-149.

Math, How to get this mathematical equation into a function? (2010). Retrieved July, 30, 2011, from http://www.excelforum.com/excel-programming/754701-howto-get-this-mathematical-equation-into-a-function.html.

Matsumoto, Y, Ino, T, Ogasawara, T. (2001). Development of Intelligent Wheelchair system with face and gaze based interface. In Proc. of the 10th IEEE Int. Workshop on Robot and Human Communication (pp. 262-267). Paris, France. Norcio A.F., Stanley J. (1989). Adaptive Human-Computer Interfaces: A Literature Survey and Perspective. IEEE Transactions on Systems, Man and Cybernetics, March, 19, no. 2, 399-408.

Okabe A., Boots B., Sugihara, K., Chiu, S. N. (2000). Spatial tessellations: concepts and applications of Voronoi diagrams. John Wiley \& Sons, British Library, 171.

Pires, G., Nunes, U. A (2002). Wheelchair steered through voice commands and assisted by reactive Fuzzy-Logic controller. Journal of Intelligent and Robotics Systems, 34, 301-314.

Reis L.P., Braga R., Sousa M., Moreira A.P., (2010). Intellwheels MMI: A Flexible Interface for an Intelligent Wheelchair, RoboCup Symposium 2009, Springer LNCS, 5949, (pp. 296-307). Graz, Austria.

Ross E. (2000). Intelligent User Interfaces: Survey and Research Directions, University of Bristol, Technical Report: CSTR-00-004 2000.

Simpson R., LoPresti E., Cooper R. (2008). How many people would benefit from a smart wheelchair?, Journal of Rehabilitation Research and Development, 45, no. $1,53-72$.

Simpson R. (2005). Smart wheelchairs: A literature review, Journal of Rehabilitation Research and Development, July/August, 423-435.

Simpson R., LoPresti E., Hayashi S., Nourbakhsh I., Miller D. (2004). The Smart Wheelchair Component System. J Rehabil Res Dev May/June; 41: no. 3B, 429442.

United Nations, (2002). Report of the Second World Assembly on Ageing. United Nations, Madrid 2002, 92-1-130221-8.

Vasconcelos, S. (2011). Multimodal Interface for an Intelligent Wheelchair, University of Porto, Faculty of Engineering. Retrieved April, 21, 2012, from http://hdl.handle.net/10216/62054. 
Vicente, S., Amaya, C., Rio, F., Balcells, A., Muniz, D. (2002). Tetranauta: an intelligent wheelchair for users with very severe mobility restrictions. Proceedings of the 2002 International Conference on Control Applications, 2, (pp. 778-783). Cancun, Mexico.

Vignier N., Ravaud J.F., Winance M., Lepoutre F.X., Ville I. (2008). Demographics of wheelchair users in France: results of national community-based handicapsincapacités-dépendance surveys, Journal of Rehabilitation Medicine, 40, 231239.

Vogel, (2008). Tongue Operated Assistive Technology, University News, 18, Issue 30, p. 14.

Wang R.H., Mihailidis A., Dutta T., Fernie G.R. (2011). Usability testing of multimodal feedback interface and simulated collision-avoidance power wheelchair for longterm-care home residents with cognitive impairments. Journal of Rehabilitation and Development, 48, 7, 801-822.

Yanco, H.A. (1998). Wheelesley: A robotic wheelchair system: Indoor navigation and user interface. In Assistive Technology and Artificial Intelligence, Applications in Robotics. User Interfaces and Natural Language Processing, Springer-Verlag, London, p. 256-268. 